\title{
Exploratory diary study of survey request frequency among research professionals
}

\author{
Kenneth E. Wallen ${ }^{1,4}$, Abbey E. Hammell ${ }^{2,4}$, and Katherine E. Dentzman ${ }^{3,4}$ \\ ${ }^{1}$ University of Idaho, Department of Natural Resources and Society, Moscow, ID USA \\ ${ }^{2}$ University of Minnesota, Liberal Arts Technologies and Innovation Services, Minneapolis, MN \\ USA \\ ${ }^{3}$ University of Idaho, Department of Agricultural Economics and Rural Sociology, Moscow, ID \\ USA \\ ${ }^{4}$ U.S. Department of Agriculture, Multistate Research Coordinating Committee and Information \\ Exchange Group: Improving Data Quality from Sample Surveys to Foster Agricultural, \\ Community, and Development in Rural America (WERA-1010)
}

\section{Author Note}

Kenneth E. Wallen (D) https://orcid.org/0000-0002-7535-5805

Abbey E. Hammell (D) https://orcid.org/0000-0002-4054-0998

Katherine E. Dentzman (D) https://orcid.org/0000-0003-3716-3578

Correspondence concerning this article should be addressed to Kenneth E. Wallen, Department of Natural Resources \& Society, University of Idaho, 875 Perimeter Drive MS 1139 Moscow, ID 83844-1139. E-mail: wallenk3@gmail.com 


\section{Abstract}

2 The survey commons is a global common-pool resource that consists of all populations of

3 potential survey participants that researchers share. As survey research methodology and

4 technology advances, the survey commons has become more accessible and used by an ever-

5 growing variety of professional and non-professional researchers, which creates a climate for

6 potential survey participants of seemingly constant requests to complete various types of

7 questionnaires. Among other factors, the frequency of survey requests likely influences burden,

8 fatigue, and data quality. Yet, few studies have explored the frequency and characteristics of

9 survey requests. In contribution to this growing research area, we conducted an exploratory

10 diary study of survey requests among research professionals for a 1-month period. Participants

11 tracked survey request dates, contact mode, response mode, title, sponsor, host, and

12 completion progress. We observed participants receive a survey request every other day, on

13 average, but only fully completed one-quarter of requests. Marketing surveys were the primary

14 request source, the main contact mode was email, and the main response mode was web-

15 based. While marketing surveys were the most frequently experienced by participants, they also

16 had the lowest completion rate; administrative and academic survey requests had the highest

17 completion rate. Overall, our exploratory diary study told an intriguing story of the quantity and

18 characteristics of survey requests among a select population and adds to growing interests in

19 survey request inquiry.

20 Keywords: deskilling, survey burden, survey climate, survey fatigue, surveys-on-surveys 


\section{Introduction}

Survey research and its associated sampling and contact methodologies have advanced

23

24

markedly over the past 75-years. The 1936 Literary Digest presidential poll, for example, ushered in considerable refinement and scientific acumen to public opinion polling (Squire, 1988). Further refinement to probability sampling followed issues associated with the 1948 Gallup presidential poll (Lusinchi, 2018). Throughout the mid-20th century, advances in telecommunications essentially made telephones ubiquitous in households, which added an additional mode of contact and response, but also complexity. The process has repeated itself with the emergence of internet and cellular technology, including email, short message services (SMS), and social media applications that now are more commonplace and trend towards ubiquity. From non-probability in-person and subscription-based mail surveys to complex, mixed-mode probability-based surveys, to rapid assessment, fit-to-purpose web-based panels, the survey research arena has evolved into an expansive enterprise that is intertwined with everyday experiences.

As modes of contact and ways to respond to surveys proliferate, some rise in favor and others fall. Take, for instance, the current proclivity of certain socio-demographic segments to communicate more via the internet or text messages, which has affected a marked decrease in random digit dialing (RDD) and other telephone-based survey responses (Dutwin \& Buskirk, 2021). That shift is exacerbated by societal trends, which show decreases in landline phone usage and increases in predatory robo and telemarketing calls to all phone types, reducing the general likelihood of any phone being answered (Lavrakas et al., 2017). This trend is evidenced by declines in RDD response rates, from $30 \%$ in the 1990 s to $9 \%$ in the 2000 s onwards (Dutwin \& Lavrakas, 2012). The internet era has seen a concomitant and exponential increase in the use of web-based surveys (Pew Research Center, 2015). Yet, despite ever-growing internet access, response rates are generally low for surveys that rely solely on an email contact to request participation (Stern, Bilgen, \& Dillman, 2014). The combination of survey response 
47 mode and response rate trends have necessitated the development of mixed-mode

48 approaches, which combine multiple contact and response modes to improve response rates

49 (Dillman et al., 2014).

50 The evolution of survey contact and response modes, in combination with the volume of

51 available contact information we share and our increasing reliance on the internet in much of the

52 world's everyday life, has facilitated an exponential expansion of survey research. Recently, that

53 expansion has been further facilitated by a deskilling trend-the process of technological

54 advancement replacing experts and allowing fewer untrained workers to complete similar

55 amounts of labor (though not necessarily equivalent or high quality (Braverman, 1998). The

56 basic tools of survey research, and the ability to develop fit-to-purpose ("plug-and-play")

57 instruments, are now available to virtually anyone with internet access (e.g., Google forms,

58 Qualtrics, QuestionPro, SurveyMonkey, and Alchemer). The ability of individuals and

59 organizations to design and implement their own surveys via low-cost online platforms allows

60 individual surveyors to contact thousands of individuals by email with survey requests that cost

61 little more than a traditional mail or phone survey of a few hundred people. The electronic

62 collection of data also makes it possible to process response data at little cost. Never before

63 have large-scale surveys been implemented so inexpensively, with all steps of data collection

64 able to be undertaken by a single individual.

65 This reality, in parallel with omnipresent marketing and advertising, builds a sense that

66 the people who comprise the populations we target for survey participants-a common-pool

67 resource shared by all who use surveys-is potentially overused, overburdened, and fatigued

68 (Olson, 2014; Leeper, 2019). Empirically, studies have shown that survey fatigue, the

69 cumulative burden placed on respondents contacted multiple times, can have negative impacts

70 on response validity (Asiu, Antons, \& Fultz, 1998), attitude towards surveys (Goyder, 1986),

71 refusal rates (McCarthy, Beckler, \& Qualey, 2006), and feelings of social contract obligation

72 (Porter, Whitcomb, \& Weitzer, 2004). Surveys perceived as invasive, lengthy, or useless are 
73 particularly fatiguing and can have a strong impact on subsequent responses to survey

74 requests, either generally or specific to a survey sponsor. Together with informal observations,

75 research suggests we may be approaching a watershed moment in the survey research field 76 where practices must be reformed or risk becoming so ineffective that the methodology is no

77 longer useful. To sectors in private industry advertising or marketing, these concerns may not

78 be particularly salient or concerning, but for others in the nonprofit, governmental, political, or

79 academic arena, these concerns are crucial to data validity, decision-making, and the relevance

80 of their profession.

81 Generally, these trends are to the detriment of the cornerstones of survey research and

82 the overall survey climate (Grove \& Couper, 1998; de Leeuw, Hox, \& Dillman, 2008; Loosveldt \&

83 Joye, 2016). In the current era of surveys, an individual is likely included in numerous sample

84 frames for probability-based surveys but also a potential convenience sample respondent for in-

85 person, mail, phone, email, text/push notification, or various other requests in the form of

86 customer receipts, websites landing pages or pop-ups, and customer feedback terminals.

87 Survey requests can come from a spectrum of illegitimate to legitimate public opinion

88 researchers, pollsters, marketers, academics, etc. using single or multi-question, short to long-

89 form opinion, feedback, or evaluative survey instruments. The state of the survey commons is

90 influenced by the number administered, their perceived legitimacy, and, importantly, the burden

91 or fatigue experienced by respondents in the form of request frequency, source legitimacy, and

92 instrument quality; a "finite survey population can support a finite survey burden" (Olson, 2014,

93 p. 94). However, few studies have attempted to empirically describe or track the state of the

94 survey commons to ask, are we collectively surveying people to such an extent that neither the

95 surveyor nor the individuals being surveyed benefits? Or, more somberly, are we collectively

96 leading to the decline of surveys as a useful methodological tool?

97 As an initial attempt to answer this question and describe the state of the survey

98 commons, we follow the tradition of "surveys-on-surveys" research to conduct an exploratory 
99 diary study. We use a convenience sample of survey research professionals who are members

100 of WERA-1010, a U.S. Department of Agriculture Multistate Research Coordinating Committee

101 and Information Exchange Group. Over a period of 1-month, we monitor survey request

102 frequency, contact/response mode, source/sponsor, and instrument type. Our objective is to

103 add to survey researchers' understanding of how the survey commons is being used,

104 particularly in the modern survey era. As exploratory research, the objective is to by no means

105 represent the survey commons as a whole, but to demonstrate a particular method that can be

106 used to empirically observe how individuals are surveyed and the forms of data and

107 interpretations that emerge from the method.

\section{Methods}

109 Participants and Procedure

A total of 23 out of 33 WERA-1010 members volunteered to participate $(69.6 \%$ response

111 rate). Participants were 13 female and 10 male members and ranged in age from 32-75. Each

112 participant was sent instructions that included a spreadsheet (offline and web-based options) to

113 serve as a survey diary. The survey diary data was collected over the course of one month,

114 from 11 November 2019 to 13 December 2019. A prenotification email to WERA-1010 members

115 was sent on 4 November 2019 to announce the study and provide members with the option to

116 opt out. The formal invitation with instructions and personal spreadsheet hyperlink and

117 document was sent on 5 November 2019. Reminder emails were sent at the end of week one

118 and the beginnings of weeks two, three, and four. The coordinating researcher was available by

119 email to answer questions from respondents about how to complete the diary.

120 Materials and Measures

121 The survey diary contained 9 columns of information for each participant to record about

122 each survey that they received an invitation for over the course of the month: (1) date survey

123 received, (2) request type, (3) contact mode, (4) response mode, (5) sponsor organization, (6)

124 host/platform, (7) title/topic, (8) participant completion, and (9) notes. Potential responses were 
125 available via a dropdown menu for some items. Respondents were also able to enter their own

126 text on any column. View example survey diary spreadsheet here:

127 https://doi.org/10.6084/m9.figshare.14396300.v1.

128 Data Analysis

All analyses were conducted in R (R Core Team, 2021). The analysis code is present in

130 our appendix accompanying this article. Descriptive statistics were calculated for total surveys

131 received, survey contact mode type, survey response mode type, survey type (i.e., sponsor)

132 category, and survey completion status. . Initial contact and response modes were provided to

133 participants as predetermined categories—email, internet, mail, phone, in-person, other-within

134 the diary, but additional categories emerged from participants' experience such that additional

135 categories were added: receipt, kiosk, web pop-ups. Barring basic descriptive statistics, we also

136 examined 1) survey type (i.e., sponsor) category by contact mode type, 2) survey type (i.e.,

137 sponsor) category by response mode type, 3) survey type (i.e., sponsor) category by completion

138 status. Additionally, we calculated the percentage that each individual participant contributed to 139 contact mode types, response mode types, and survey type (i.e., sponsor) categories that were 140 reported.

141 Categorizing Survey Type

142 Survey type categories were developed via an inductive process by the researchers, as

143 the diary did not provide a preselected category for the survey type. Data suggested a survey

144 typology based on the survey sponsor organization and survey topic/title. We created a typology

145 of five survey request types: academic, administrative, political, marketing, or unknown.

146 Academic surveys were defined as surveys that had a scholarly research focus, e.g.,

147 surveys addressing the effectiveness of educational websites, fishing attitudes and behaviors,

148 scientists' public engagement activities, female faculty experiences in agricultural education,

149 and bladder health. Administrative surveys were defined as surveys that were solicited from a

150 group or organization the respondent belonged to, e.g., a university or professional 
151 organization. Examples included requests for input on changing the name of an academic

152 conference, research priorities at a university, satisfaction with campus services, support for a

153 campus smoking ban, and an organizational t-shirt logo contest. Political surveys came from

154 various listservs respondents were subscribers of and, generally, were specific to a political

155 candidate. Marketing surveys requested feedback on experiences, services, products, and other

156 consumer-related activities, and were the most common and varied category. Examples ranged

157 from mundane post-service satisfaction surveys from hotel chains and airlines to health

158 services, e.g., satisfaction with a recent surgery and non-topical pre-appointment questionnaires

159 at an urgent care clinic, to survey requests on receipts or after online purchases. Finally,

160 surveys that did not have a clear purpose were listed as unknown; respondents noted that these

161 surveys seemed suspicious because no topic was specified.

\section{Results}

The 23 participants received a total of 314 survey requests over the 1 -month period of

164 the study, with an average of 13.65 survey requests per person $(S D=8.77)$. The lowest

165 number of survey requests received by a participant was 1 and the highest 33 . The median

166 number of surveys was 12, and the mode was 12 and 13 (three participants each).

\section{Survey Contact Mode}

Email was the predominant contact (invitation) mode, with $74.85 \%(n=235)$ of survey

169 requests coming via email. The second most frequent was receipts $8.60 \%(n=27)$, followed by

170 internet sites $6.69 \%(n=21)$. All remaining modes, individually, made up less than $3 \%$ of the

171 requests (see Table 1$)$. 
Table 1

Contact Mode

\begin{tabular}{lcc}
\hline & Frequency & Percent \\
\hline Email & 235 & 74.85 \\
Receipt & 27 & 8.60 \\
Internet & 25 & 7.96 \\
$\quad$ General Internet & 21 & 6.69 \\
$\quad$ Pop-up & 4 & 1.27 \\
Phone & 8 & 2.55 \\
In-person & 7 & 2.23 \\
Mail & 7 & 2.23 \\
Text (SMS) & 2 & 0.64 \\
Kiosk & 1 & 0.32 \\
Other & 2 & 0.63 \\
\hline
\end{tabular}

\section{Survey Response Mode}

$174 \quad$ For response mode, $75.79 \%(n=238)$ of surveys requested responses via filling out an

175 online form (internet website). Approximately $16.24 \%(n=51)$ of surveys requested responses

176 by email, and less than $2 \%$ of surveys requested responses to be given by mail $(n=6)$, phone

$177(n=6)$ or receipt $(n=5$; see Table 2$)$. All remaining modes, individually, made up less than $1 \%$ 178 of the response mode requests (see Table 2). 
Table 2

Response Mode

\begin{tabular}{lcc}
\hline & Frequency & Percent \\
\hline Internet & 238 & 75.79 \\
Email & 51 & 16.24 \\
Mail & 6 & 1.91 \\
Phone & 6 & 1.91 \\
Receipt & 5 & 1.59 \\
In-person & 1 & 0.32 \\
Text (SMS) & 1 & 0.32 \\
Tablet & 1 & 0.32 \\
Other & 5 & 1.59 \\
\hline
\end{tabular}

180 Individual Contributions to Survey Contact \& Response Modes

181 Overall, we found online survey contact and response requests to be the most widely

182 experienced by our participants. In particular, all of our participants experienced at least one

183 survey contact request online-- all but one of the participants were contacted via email at least

184 once $(95.65 \% ; n=22)$, and the remaining person (Participant 13) was contacted through the

185 internet, generally. Eight (34.78\%) of the participants were contacted through the internet,

186 generally (pop-ups included) at least once. After online contact modes, the contact modes

187 experienced by the most participants were receipts $(30.43 \%, n=7)$ and mail $(30.43 \%, n=7)$.

188 Figure 1 below shows the percentage that each individual participant contributed to each

189 contact mode request type. 


\section{Figure 1}

191 Individual Contributions to Total Contact Mode Requests

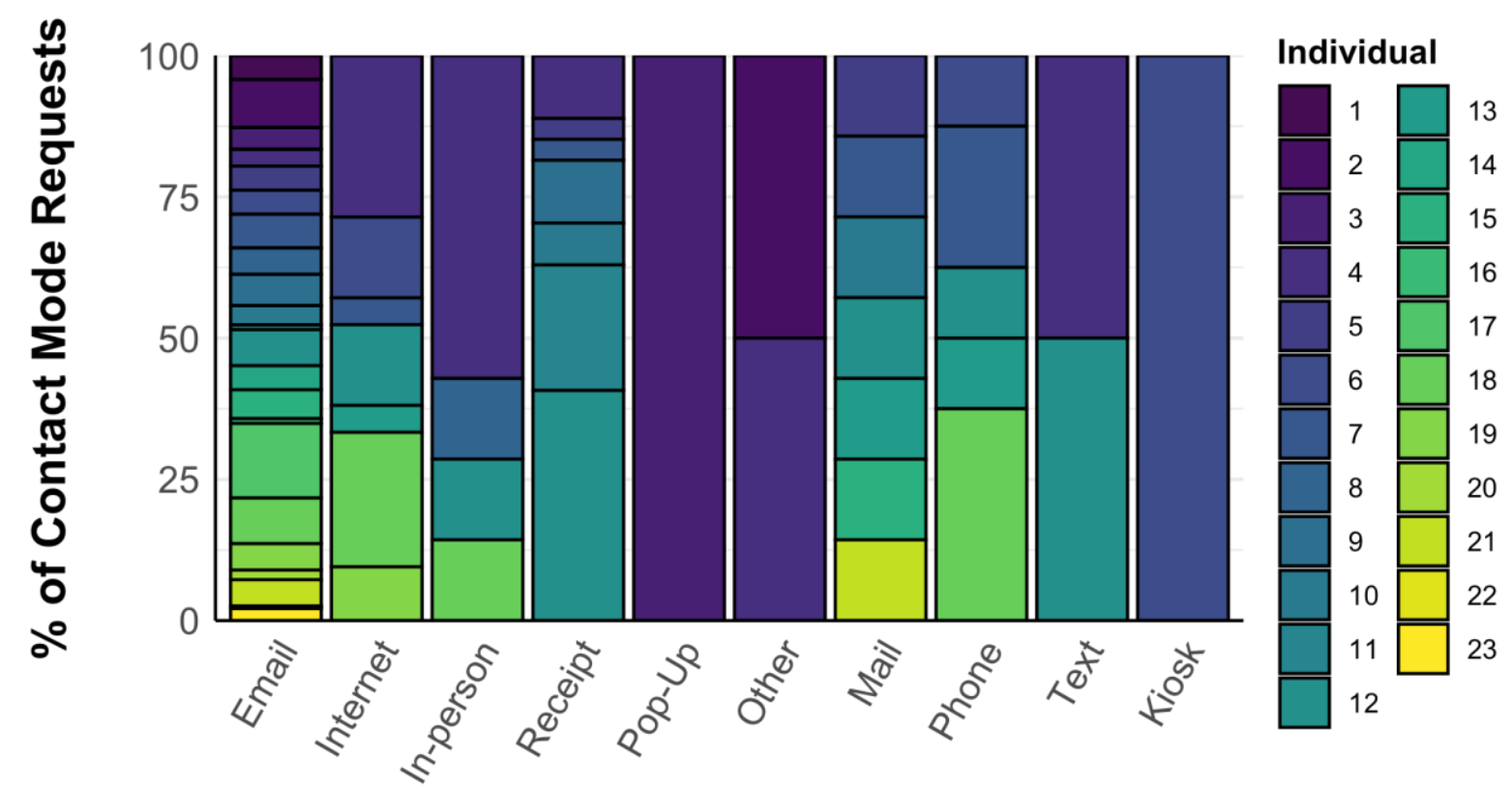

\section{Contact Mode}

193 Note. Shows the percentage of survey requests each individual contributed for each contact 194 mode request type.

Similar to contact modes, all of our participants experienced at least one survey

196 response mode request online-- all but two of the participants were asked to complete at least

197 one survey via an online platform (e.g., Qualtrics, Survey Monkey, etc.; $91.30 \% ; n=21$ ), and

198 the remaining two individuals (Participants 17 and 21) were asked to complete at least one

199 survey via email. Five of the participants were asked to respond to at least one survey via email

200 (21.74\%). After online response modes, the response mode requests experienced by the most

201 participants were mail $(26.09 \%, n=6)$ and email $(21.74 \%, n=5)$. Figure 2 shows the

202 percentage that each individual participant contributed to each response mode request type. 
Figure 2

204 Individual Contributions to Total Response Mode Requests

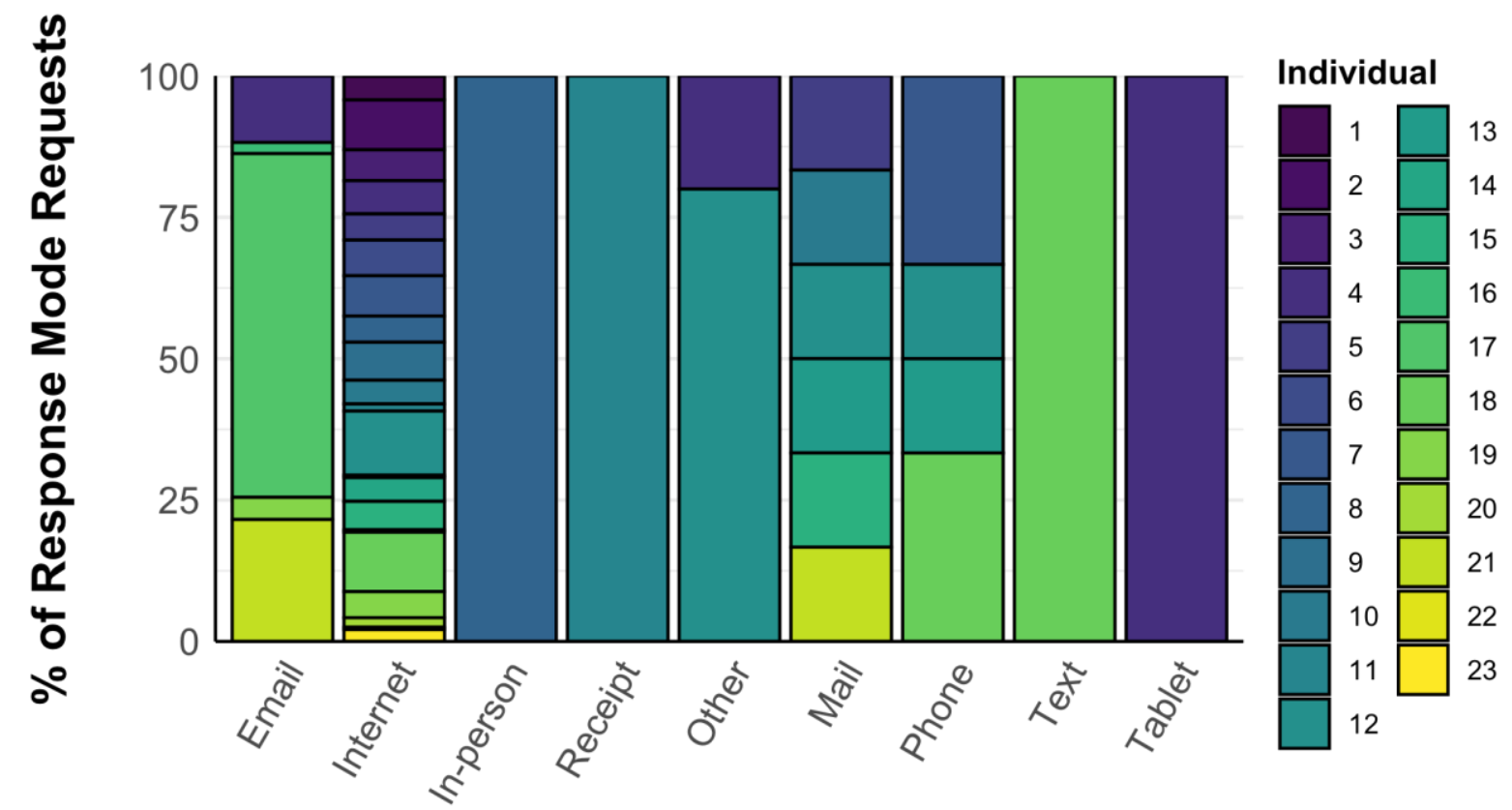

Response Mode

206 Note. Shows the percentage of survey requests each individual contributed for each response 207 mode request type.

\section{Survey Type Count}

The three authors each individually categorized all survey diary entries into one of the

210 five categories. All coders agreed on the survey type categorization for $83 \%$ of diary entries

211 during the first round of survey type coding (Krippendorff's alpha $=.82$ ). At least two of three

212 coders agreed on the survey type categorization for all entries, which provided a consensus

213 approach to finalize categorization. Out of all 314 survey requests, we observed that $47.13 \%$ ( $n$

$214=148)$ were marketing surveys, $30.89 \%(n=97)$ were administrative surveys, $8.28 \%(n=26)$

215 were political surveys, $4.14 \%(n=13)$ were academic surveys, and $9.55 \%(n=30)$ were an

216 "unknown" survey type. 


\section{Individual Contributions to Survey Type Categories}

We were interested in how much any specific person contributed to the number of total

219 surveys in each survey type category to 1) determine how much our results for each survey type

220 category may be influenced by any one individual and 2) determine if different survey type

221 requests were experienced by many, or just a few, of our participants. Figure 3 shows the

222 percentage of survey requests that each individual participant contributed to each survey type

223 category.

224 We found administrative and marketing survey invitations to be the most universally

225 received among our participants, with $95.65 \%(n=22)$ of respondents receiving at least one

226 administrative survey invitation and $86.96 \%(n=20)$ of participants receiving at least one

227 marketing survey invitation. On the other hand, only $30.43 \%(n=7)$ of our participants received

228 an academic survey invitation, and an even smaller percentage of our respondents received a

229 political survey invitation $21.74 \%(n=5)$. Approximately $65.22 \%(n=15)$ of our participants

230 received survey invitations that fell outside of our four main survey type categories, into the

231 "undefined" category. 


\section{$232 \quad$ Figure 3}

233 Individual Contributions to Total Survey Type Requests

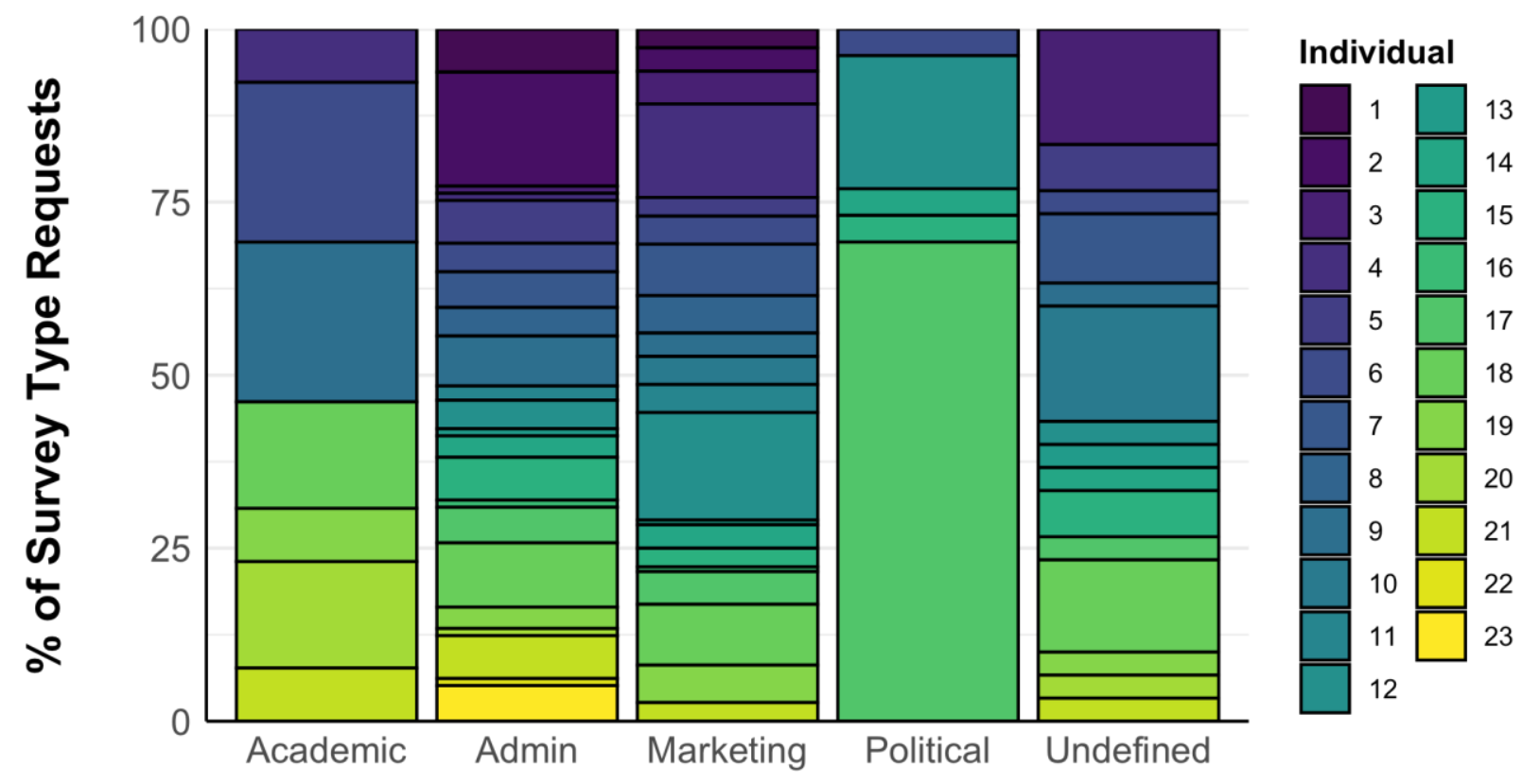

\section{Survey Type}

235 Note. Shows the percentage of survey requests contributed by each individual participant for 236 each survey type category.

\section{Survey Type Contact Modes}

$238 \quad$ For all survey types, email was the primary contact mode experienced by our 239 participants. In particular, $93.81 \%(n=91)$ of administrative surveys, $58.11 \%(n=86)$ of 240 marketing surveys, $96.15 \%(n=25)$ of political surveys, $84.62 \%(n=11)$ of academic surveys, 241 and $73.33 \%(n=22)$ of undefined surveys received had contact requests over email. For all 242 survey types besides marketing, all other contact modes made up equal to or less than $10 \%$ of 243 the contact request modes for a given survey type (see Figure 4). Marketing had the most

244 diverse contact mode types, with receipts $(18.24 \%, n=27)$ and internet $(12.17 \%, n=18$, pop-

245 ups included) also making up a decent percentage of contact requests. All other contact mode 
246 types for marketing surveys made up less than $5 \%$ of the total marketing survey contacts (see

247 Figure 4 and Appendix).

\section{$248 \quad$ Figure 4}

249 Contact Modes by Survey Type

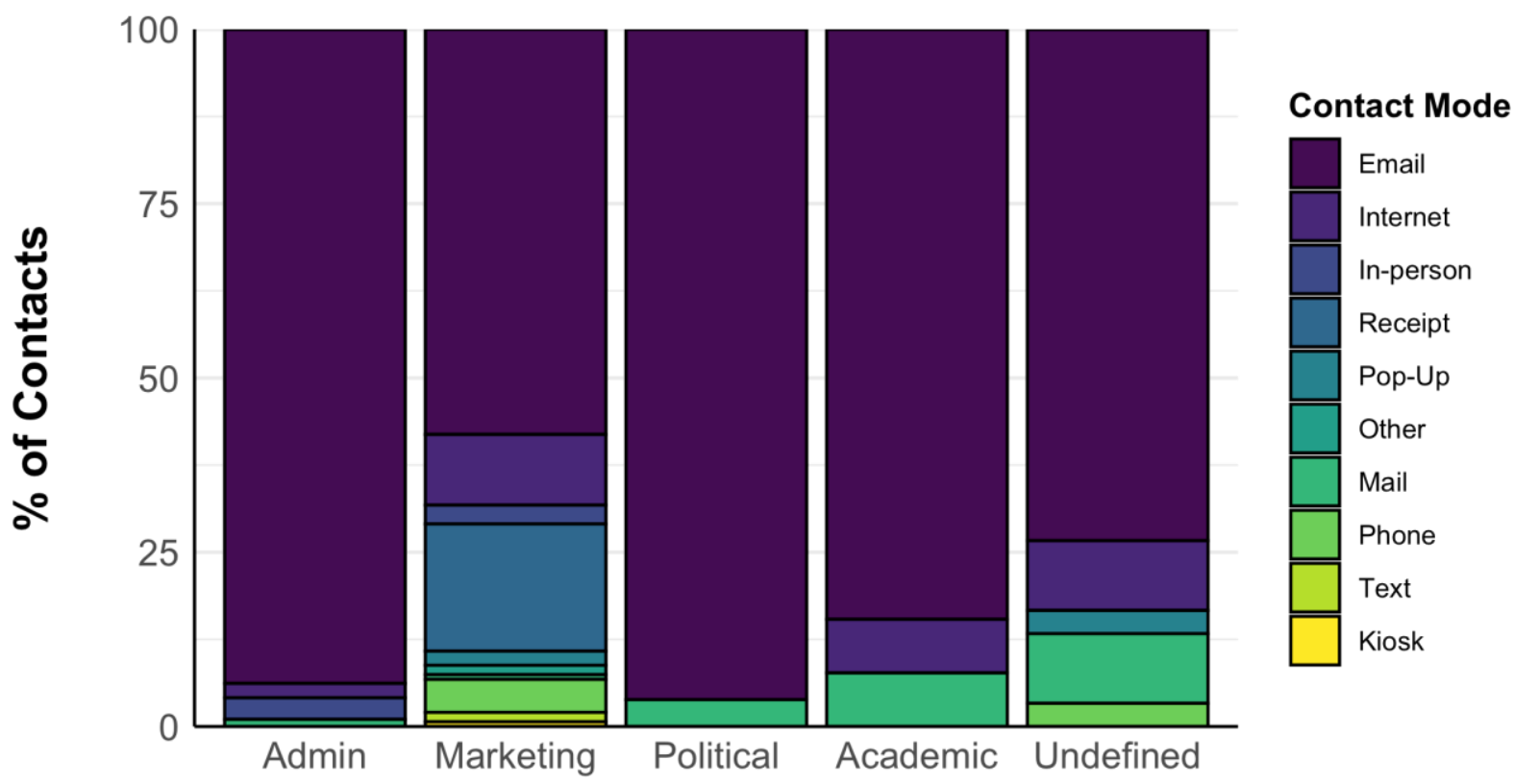

251 Note. Shows the percentage of contact modes used for each survey type category. A majority of contacts made for each survey type category were through email.

\section{Survey Type Response Modes}

For all survey type categories besides political, responding through an internet platform

255 was the primary response mode experienced by our participants. In particular, $84.54 \%(n=82)$

256 of administrative surveys, $75.68 \%(n=112)$ of marketing surveys, $92.31 \%(n=12)$ of academic

257 surveys, and $83.33 \%(n=25)$ of undefined surveys requested that participants respond over the

258 internet. On the other hand, a majority of political surveys asked for responses over email

$259(69.23 \% ; n=18)$, followed by the internet $(26.92 \% ; n=7)$. Email response requests also made 
260 up a decent amount of response requests for both administrative $(12.37 \%, \mathrm{n}=12)$ and

261 marketing $(12.84 \%, n=19)$ surveys. Besides the response modes outlined above, all other

262 response mode requests made up less than $8 \%$ of the contact request modes for a given survey 263 type (see Figure 5 and Appendix).

\section{Figure 5}

265 Response Modes by Survey Type

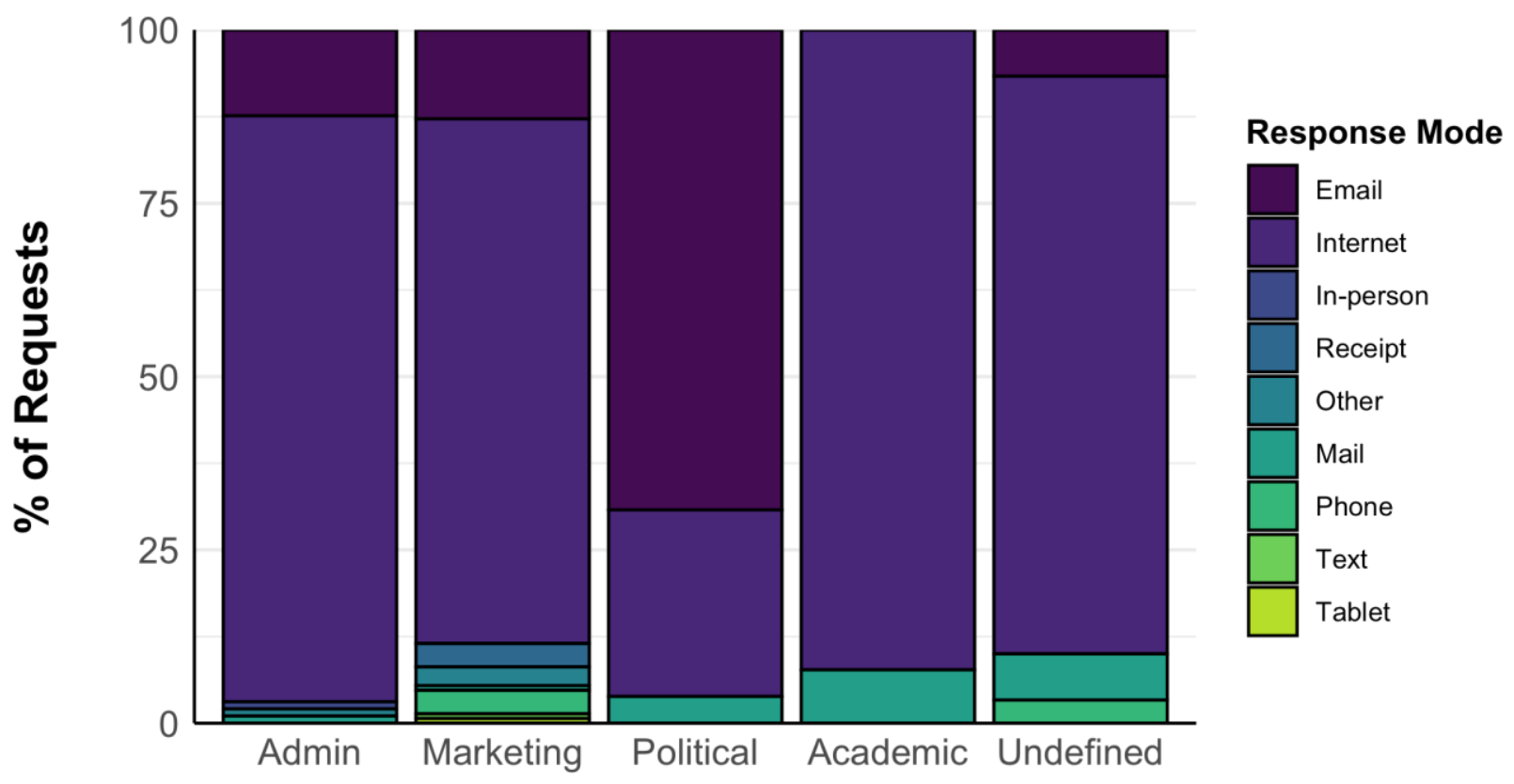

\section{Survey Type}

267 Note. Shows the percentage of response mode requests for each survey type category. A vast 268 majority of response modes requested for each survey type category were internet or email.

\section{Survey Completion}

270 Approximately $4.46 \%$ (14 out of 314 ) surveys did not have completion status reported.

271 By survey type, 6.67\% (2 out of 30 ) undefined surveys did not have completion status reported, $2725.41 \%$ (8 out of 148 ) of marketing surveys did not have completion status reported, $3.85 \%$ (1 273 out of 26) of political surveys did not have completion status reported, and $3.09 \%$ (3 out of 97 ) 
274 of administrative surveys did not have completion status reported. All academic surveys had a 275 completion status reported.

$276 \quad$ Figure 6 shows completion distribution by survey type category. Of the 300 surveys 277 where completion status was reported, approximately $68.33 \%$ percent $(n=205)$ of surveys were 278 not completed, even partially, $26.0 \%$ percent $(n=78)$ of surveys were completed fully, and $6.0 \%$ $279(n=18)$ of surveys were partially completed. Of the surveys where completion status was 280 reported, administrative surveys were the most completed, with $47.87 \%$ (45 out of 94 ) of them 281 being fully completed, followed closely by academic surveys (46.15\%; 6 out of 13 ), and more 282 distantly by marketing (12.14\%; 17 out of 140$)$ and political surveys (12.0\%; 3 out of 25$)$.

\section{$283 \quad$ Figure 6}

284 Completion by Survey Type

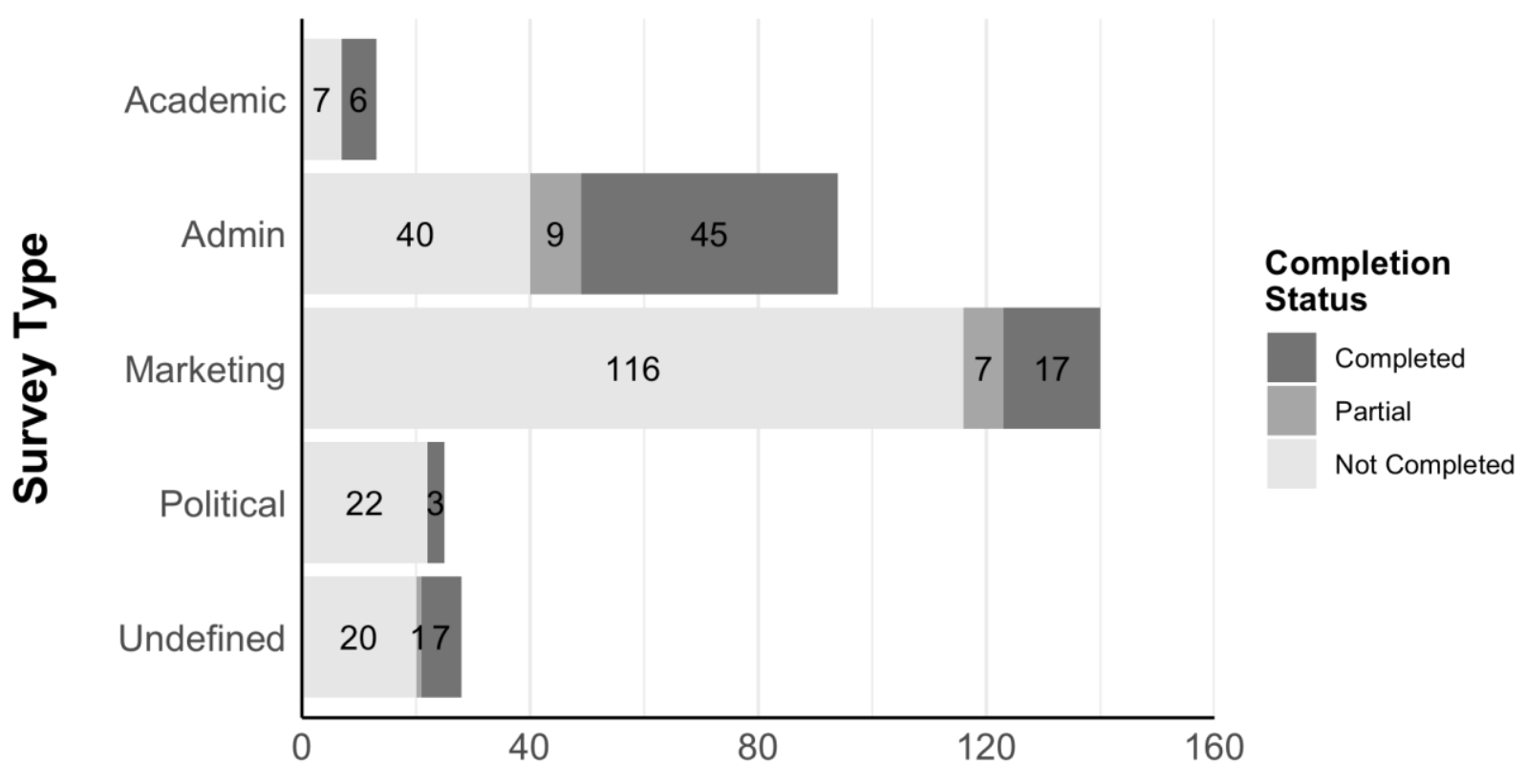

\section{Number of Surveys}

286 Note. Shows the total number of surveys within each survey type category that were completed, 287 partially completed (i.e., partial), or not completed. Surveys that did not have a completion 288 status reported $(n=14)$ by the participant are excluded from this graph. 
Figure 7 shows percent of surveys that were not completed, even partially, by survey

290 type category; $88.0 \%$ (22 out of 25 ) of political survey requests received were not completed,

291 followed closely by marketing surveys (82.86\%; 116 out of 140 ), and more distantly by

292 academic surveys (53.85\%; 7 out of 13 ) and administrative surveys (42.55\%; 40 out of 94 ).

\section{$293 \quad$ Figure 7}

294 Percent of Surveys Not Completed, by Survey Type

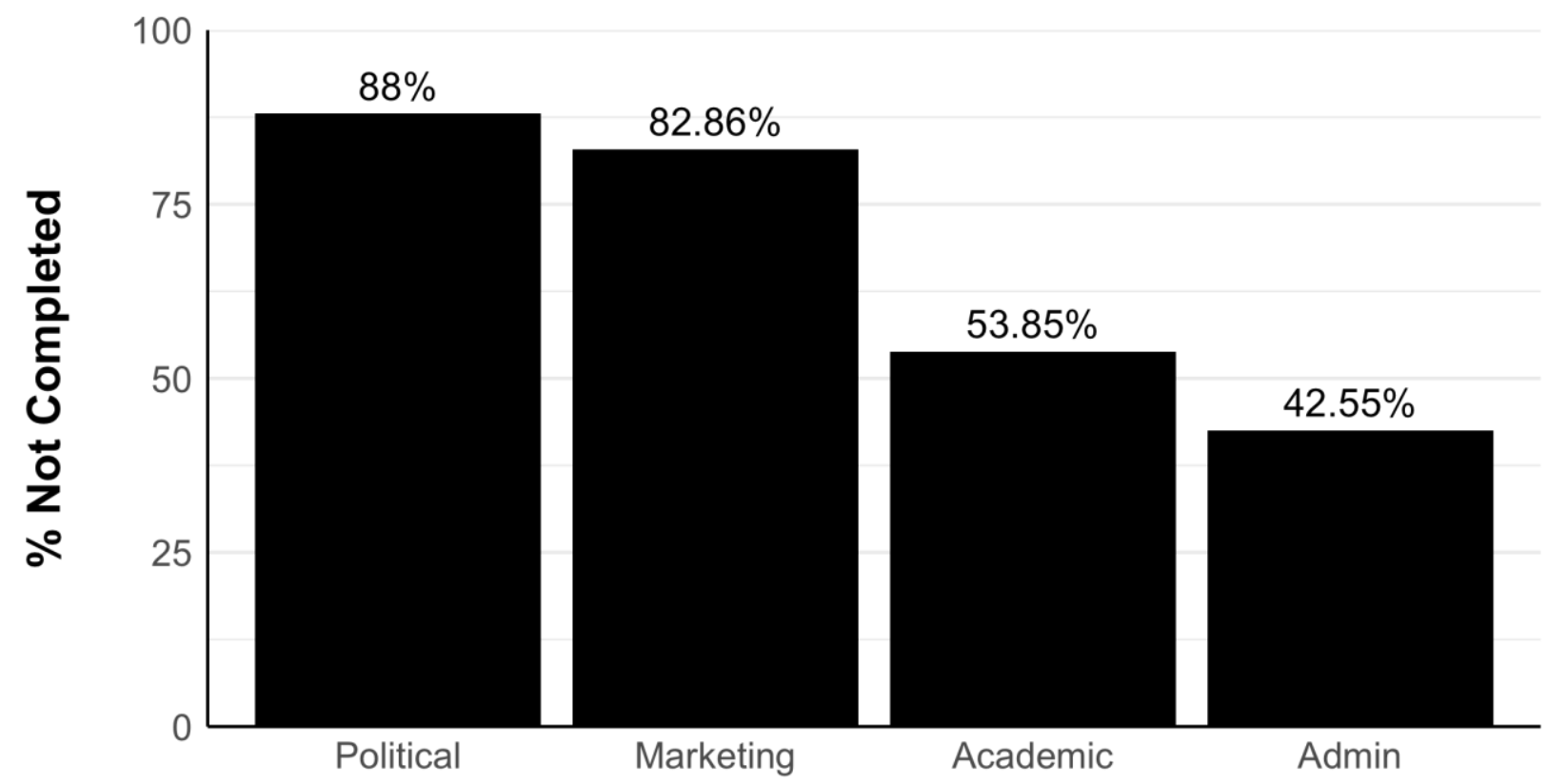

296 Note. Shows the overall percentage of total surveys that were not completed, even partially, by

297 survey type category. Surveys that did not have a completion status reported $(n=14)$ by the 298 participant are excluded from this graph.

\section{Discussion}

This exploratory study examined the quantity, frequency, and characteristics of survey

301 requests, such as contact and response mode, source and sponsor, and instrument type, via a 302 self-report diary method for a 1-month period among a convenience sample of survey research 
303 professionals. We observed these individuals receive 12-13 survey requests over a 1-month

304 period and that these requests are primarily from a marketing source/sponsor, the main contact

305 mode type is email, and the main response mode type is web-based. Examining contact and

306 response modes by survey (i.e., sponsor) type category showed the ubiquity of email contact

307 and web-based (internet or email) response modes across all survey type categories. Though

308 marketing surveys were the most frequently reported survey type, they also had the lowest

309 completion rate among the sample. Administrative and academic survey requests, whose

310 cumulative percentage was lower than marketing requests, nonetheless, had the highest

311 completion rate. However, this high completion rate of administrative and academic surveys

312 could be a systematic bias given the sample was all survey researchers/professionals. Perhaps

313 unsurprising in consideration of the downward trend of response rates (Tourangeau \& Plewes,

314 2013), over two-thirds of survey requests went unheeded, even among a sample of survey

315 professionals. In terms of individual differences, we observed a large majority of participants

316 received at least one marketing and administrative survey request, but only a smaller portion of

317 our sample received at least one academic and political survey request over the course of the

318 month. Overall, our exploratory diary study tells an intriguing story that warrants further

319 investigation either via similar scale replicates or larger scale application among various 320 populations.

From our main objective to investigate survey request frequency and type, findings

322 suggest our sample exists in a state of constant survey requests, receiving, on average, 12-14

323 requests in the study's one-month period. However, it is a subjective interpretation to conclude

324 that a population is overused, overburdened, and experiencing survey fatigue (Olson, 2014;

325 Leeper, 2019). What people define or recognize as a survey request, and the extent to which

326 their attitude towards that request frequency is positive or negative, is likely highly individualized

327 and relative to circumstance and experience. Recently, de Leeuw, Hox, Silber, Struminskaya, 
and Vis (2019) developed a survey attitude scale that may add an explanatory covariate or complementary dimension of understanding to survey request frequency research.

Relatedly, we did not ask participants to complete each survey requested or to record

331 the survey length and their overall experience with the survey if they did. Because of this, we do

332 not have a specific understanding if any survey was perceived as invasive, lengthy, or useless.

333 As we note, research and survey research professionals are beginning to question which

334 practices, if any, should or can be reformed to address survey commons issues. Private sectors

335 may also share these concerns, but the relationship between public, nonprofit, and private

336 survey, polling, and marketing researchers is not well facilitated. This might be expected given

337 the ubiquity of marketing messages and advertisements in Americans lives, and receptivity to them (American Association of Advertising Agencies, 2007; Media Dynamics, 2014), marketing surveys were the most frequent request received by participants but also the least completed.

340 But unlike research into the frequency of daily marketing messages and advertisements

341 experienced by individuals, survey request frequency is a relatively new domain of inquiry, and

342 no baseline research exists with which to compare our results. Whether via diary methods or 343 more traditional methods, our results, because interpretation from an exploratory study of an

344 opportunistic sample, suggest needed inquiry at the intersection of survey frequency, subjective 345 burden, and receptivity (i.e., general survey attitude), either via additional exploratory or more 346 systematic designs. It would also be beneficial to further refine survey typologies (e.g.,

347 "academic", "administrative", "marketing", etc.) and determine how survey typology may interact 348 with subjective burden and receptivity.

349 Contact and response mode results were consistent with general trends of web-based 350 surveys becoming the dominant form (European Society for Opinion and Marketing Research, 351 2018). Moreover, the accessibility, user-friendly operations, and do-it-yourself ethos of online 352 survey platforms, likely plays a role in the predominance of web and email, regardless of survey 353 type. These characteristics of online survey platforms can make it easier to create and 
354 administer an online survey quickly, without needing to consult a survey professional. Given the

355 survey research issues of the 21st century, telephone and mail surveys must now be

356 implemented on a well-funded project in a skillful and strategic manner, based on several

357 factors associated with the target population, question types, budget, timeline, and other

358 logistics. In contrast, web-based contact and survey instruments appear to be a general default

359 (though many considerations should be considered to determine an appropriate contact and

360 response mode). Given that most online survey platforms are (a) developed and marketed for

361 questionnaire design rather than research or sample design and (b) relatively cheaper to

362 implement, it seems logical their user and logistics friendly nature would facilitate prevalence.

363 Although our sample results show a dominance of web-based surveys and contact, it would be

364 valuable for future research to determine how different demographic characteristics and life experiences of individuals may affect the modes of the survey requests that they receive.

Survey completion rate, though not a direct research objective of the survey diary, was

367 an interesting finding. Considering the study sample was survey research professionals, one

368 might assume fewer refusals and less reluctance to complete any given survey. However, from

369 a diversity of survey types, modes, sponsors, and, presumably, lengths, over two-thirds of

370 surveys were not completed by our sample. Moreover, only half of administrative and academic

371 surveys were completed, which are the sectors in which all participants primarily work. Perhaps,

372 not surprisingly, marketing and political surveys were of the least interest to participants. Yet,

373 these are the sectors that have the infrastructure and capacity to inundate the survey commons

374 with repeated and differentiated survey requests. From that, combined with the trends of high

375 refusal and low survey completion observed in our study, we may surmise that the survey

376 commons could presumably suffer with coinciding response, cooperation, contact, and

377 completion rate decreases (Krosnick, Presser, Fealing, Ruggles, \& Vannette, 2015). Though, it

378 should be noted that while this sample completed approximately half of the academic and

379 administrative surveys, only $\sim 20 \%$ of political and marketing surveys were completed. 
380 Seemingly, participants differentiate between survey type (i.e., sponsor) categories that were 381 worth their time, which suggests a varied attitude towards surveys dependent on the survey 382 sponsor type (de Leeuw et al., 2019).

383 Limitations and Future Research

The extent to which these findings represent the survey commons, as a whole, is not an appropriate interpretation, but our results are consistent with empirical and anecdotal trends 386 (Couper, 2017; Krosnick et al., 2015). That is, we cannot draw particularly strong conclusions 387 beyond our study's parameters, given the convenience and homogeneity of the sample, but do 388 consider them within the scope of conventional wisdom concerning contemporary issues 389 affecting the survey climate and the survey commons (e.g., Leeper, 2019; Olson, 2014; Porter 390 et al., 2004). Future research may employ more systematic methodologies to achieve results 391 with more generalizability. Relatedly, while one of our working assumptions was that deskilling 392 influences survey request frequency, we were unable to determine the extent to which that 393 influences survey fatigue or the number of deskilled surveys (i.e., plug-and-play/do-it-yourself 394 questionnaire design and implementation by untrained/under-trained researchers). Research designs and methodologies other than a diary study are better suited to address these assumptions directly.

An important limitation and avenue for future research is the fact that awareness of a 398 survey request is contingent upon what one considers a survey and a request. For instance, do 399 minimally invasive or passive requests on a receipt or presented on a kiosk contribute to survey 400 burden or negatively affect the survey commons? Are they considered a request? The same 401 question might be asked about bothersome but easily ignored product feedback survey 402 requests one receives for a product they purchased or an event they attended. We developed 403 our own survey typology through a standard inductive process, but future studies might consider 404 allowing participants to self-categorize the survey requests they receive given their perceptions 405 of the request and associated burden is the essential element to understand any issues 
associated with the survey commons. Likewise, providing participants with examples of what the researchers view as survey requests and allowing participants to render their opinion on whether they consider them requests is another consideration. In addition to allowing participants to contribute to survey type categorization, future research may also consider research designs and data collection instruments that facilitate examination of differences in the

411 types of survey requests that individuals receive based on demographic characteristics and

412 other relevant segmentation variables. In general, limitations imposed by the study design and

413 our choice of analytical procedures had significant bearing on what questions could be

414 answered and how findings could be interpreted.

\section{Conclusion}

416 As exploratory inquiry, our findings and interpretations are admittedly limited to the 417 parameters of the cross-sectional study design and convenience sample. Yet, results are 418 intriguing and point towards a novel and salient line of inquiry that is a growing research 419 imperative for the future of survey research and our shared survey commons. That inquiry is 420 one that considers not just the gross frequency of survey requests, but the net, based on a 421 typology of survey requests that aligns with current practices and technologies, each of which 422 may pattern receptivity and participation in distinct ways. Individual and group differences in 423 refusal and completion rates by mode and demographics, while known to some extent in 424 generalities, may become more nuanced and predictive when request frequency is considered. 425 Overall, the diary method was a useful method to achieve our objectives and provide answers to 426 our research questions while simultaneously opening the door to more questions. In conclusion, 427 we hope future research can use all or parts of the framework we have provided here to 428 examine the landscape of survey requests and completion rates. We anticipate and encourage 429 survey research practitioners and methodologists to pursue survey request frequency 430 investigations and view our findings as facilitating that pursuit. 
We thank all past and current members of WERA-1010: Improving Data Quality from

433 Sample Surveys to Foster Agricultural, Community, and Development in Rural America, a U. S.

434 Department of Agriculture Multistate Research Coordinating Committee and Information

435 Exchange Group. We thank our participants for their diligence and attention to detail during the

436 duration of the diary study.

\section{Supporting Information}

438 Appendix includes an outline of our data analyses in R (https://doi.org/10.6084/m9.figshare.

439 15907551). Analysis code in R is also available as an R Markdown file (https://doi.org/

$440 \quad 10.6084 / \mathrm{m} 9$. figshare.15910749). The survey diary spreadsheet template is available

441 (https://doi.org/10.6084/m9.figshare.14396300.v1). The authors are solely responsible for the

442 content and functionality of these materials. Queries (other than absence of the material) should

443 be directed to the corresponding author.

Literature Cited

445 American Association of Advertising Agencies. (2007, Feb 15). How many advertisements is a person exposed to in a day? https://ams.aaaa.org/eweb/upload/fags/adexposures.pdf

447 Asiu, B. W., Antons, C. M., \& Fultz, M. L. (1998). Undergraduate perceptions of survey participation: Improving response rates and validity. Association for Institutional Research Annual Forum Paper, 1-15. https://files.eric.ed.gov/fulltext/ED422805.pdf

Braverman, H. (1998). Labor and monopoly capital: The degradation of work in the twentieth century. New York University Press.

452 Couper, M. P. (2017). New developments in survey data collection. Annual Review of Sociology, 43, 121-145. https://doi.org/10.1146/annurev-soc-060116-053613

454 de Leeuw, E. D., Hox, J. J., \& Dillman, D. A. (Eds.). (2001). International handbook of survey 455 methodology. Routledge.

456 de Leeuw, E., Hox, J., Silber, H., Struminskaya, B., \& Vis, C. (2019). Development of an 457 international survey attitude scale: Measurement equivalence, reliability, and predictive 
validity. Measurement Instruments for the Social Sciences, 1, 1-10. https://doi.org/10.1186/s42409-019-0012-x

Dutwin, D., \& Buskirk, T. D. (2021). Telephone sample surveys: dearly beloved or nearly departed? Trends in survey errors in the era of declining response rates. Journal of Survey Statistics and Methodology, 9(3), 353-380. https://doi.org/10.1093/issam/smz044

Dutwin, D., \& Lavrakas, P. (2016). Trends in telephone outcomes, 2008 - 2015. Survey Practice, 9(3). https://doi.org/10.29115/SP-2016-0017

European Society for Opinion and Marketing Research. (2018). Global market research report 2018: An ESOMAR industry report.

Goyder, J. (1986). Surveys on surveys: Limitations and potentialities. Public Opinion Quarterly, 50(1), 27-41. https://doi.org/10.1086/268957

Groves, R., \& Couper, M. (1998). Nonresponse in household surveys. John Wiley \& Sons.

470 Krosnick, J. A., Presser, S., Fealing, K. H., Ruggles, S., \& Vannette, D. L. (2015). The future of survey research: Challenges and opportunities. National Science Foundation Advisory Committee for the Social, Behavioral and Economic Sciences. https://www.nsf.gov/sbe/AC Materials/The Future of Survey Research.pdf

Lavrakas, P., et al. (2017). The future of U.S. general population telephone survey research. American Association for Public Opinion Research. https://www.aapor.org/EducationResources/Reports/The-Future-Of-U-S-General-Population-Telephone-Sur.aspx

Leeper, T. J. (2019). Where have the respondents gone? Perhaps we ate them all. Public Opinion Quarterly, 83, 280-288. https://doi.org/10.1093/pog/nfz010

Loosveldt, G., \& Joye, D. (2016). Defining and assessing survey climate. In C. Wolf, D. Joye, T. W. Smith, \& Y. Fu (Eds.), The Sage handbook of survey methodology (pp. 67-76). Sage. https://dx.doi.org/10.4135/9781473957893.n6 
482 Lusinchi, D. (2018). "The Great Fiasco" of the 1948 presidential election polls: status recognition and norms conflict in social science. Annals of Science, 75, 120-144. https://doi.org/10.1080/00033790.2018.1466194

McCarthy, J. S., Beckler, D. G., \& Qualey, S. M. (2006). An analysis of the relationship between survey burden and nonresponse: If we bother them more, are they less cooperative? Journal of Official Statistics, 22(1), 97.

Media Dynamics. (2014). America's media usage Trends and ad exposure: 1945-2014. https://www.mediadynamicsinc.com/uploads/files/PR092214-Note-only-150-Ads-2mk.pdf

Olson, C. (2014). Survey burden, response rates, and the tragedy of the commons. Journal of Continuing Education in the Health Professions, 34(2), 93-95. https://doi.org/10.1002/chp.21238

493 Pew Research Center. (2015, June 10). Three technology revolutions. https://www. pewresearch.org/internet/three-technology-revolutions/

495 Porter, S.R., Whitcomb, M.E., \& Weitzer, W.H. (2004). Multiple surveys of students and survey fatigue. New Directions for Institutional Research, 121, 63-73. https://doi.org/10.1002/ir.101

498 R Core Team (2021). R: A language and environment for statistical computing. R Foundation for Statistical Computing, Vienna, Austria. URL http://www.R-project.org/

500 Squire, P. (1988). Why the 1936 Literary Digest poll failed. Public Opinion Quarterly, 52, 125-

$501 \quad$ 133. https://doi.org/10.1086/269085

502 Tourangeau R., \& Plewes T. J., (Eds.) (2013). Nonresponse in social science surveys: A 503 research agenda. National Academies Press. https://doi.org/10.17226/18293 